\title{
Modified Uridines with C5-methylene Substituents at the First Position of the tRNA Anticodon Stabilize U.G Wobble Pairing during Decoding ${ }^{*[5}$
}

Received for publication, January 9, 2008, and in revised form, February 20, 2008 Published, JBC Papers in Press, May 2, 2008, DOI 10.1074/jbc.M800233200

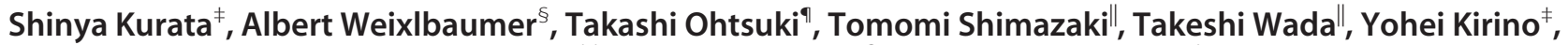
Kazuyuki Takai**, Kimitsuna Watanabe ${ }^{\ddagger \neq}$, V. Ramakrishnan ${ }^{\S}$, and Tsutomu Suzuki ${ }^{\ddagger}$

From the ${ }^{\ddagger}$ Department of Chemistry and Biotechnology, Graduate School of Engineering, University of Tokyo, 7-3-1 Hongo,

Bunkyo-ku, Tokyo 113-8656, Japan, ${ }^{\S}$ Medical Research Council, Laboratory of Molecular Biology, Hills Road,

Cambridge CB2 0QH, United Kingdom, the "Department of Bioscience and Biotechnology, Faculty of Engineering, Okayama University,

3-1-1 Tsushimanaka, Okayama 700-8530, Japan, the Department of Medical Genome Sciences, Graduate School of Frontier Sciences, Universitiy of Tokyo, Bioscience Building 702, Kashiwa, Chiba 277-8562, Japan, ${ }^{* *}$ Cell-free Science and Technology Research

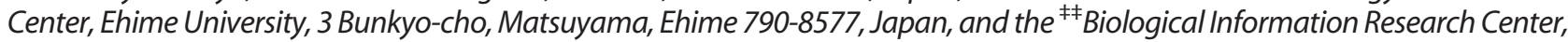
National Institute of Advanced Industrial Science and Technology, 2-42 Aomi, Koto-ku, Tokyo 135-0064, Japan

Post-transcriptional modifications at the first (wobble) position of the tRNA anticodon participate in precise decoding of the genetic code. To decode codons that end in a purine (R) (i.e. NNR), tRNAs frequently utilize 5-methyluridine derivatives $\left(\mathrm{xm}^{5} \mathrm{U}\right)$ at the wobble position. However, the functional properties of the C5-substituents of $\mathrm{xm}^{5} \mathrm{U}$ in codon recognition remain elusive. We previously found that mitochondrial tRNAs ${ }^{\text {Leu(UUR) }}$ with pathogenic point mutations isolated from MELAS (mitochondrial myopathy, encephalopathy, lactic acidosis, and stroke-like episodes) patients lacked the 5-taurinomethyluridine $\left(\tau \mathrm{m}^{5} \mathrm{U}\right)$ modification and caused a decoding defect. Here, we constructed Escherichia coli tRNAs ${ }^{\text {Leu(UUR) }}$ with or without $\mathrm{xm}^{5} \mathrm{U}$ modifications at the wobble position and measured their decoding activities in an in vitro translation as well as by A-site tRNA binding. In addition, the decoding properties of tRNA ${ }^{\text {Arg }}$ lacking $\mathrm{mnm}^{5} \mathrm{U}$ modification in a knock-out strain of the modifying enzyme $(\Delta m n m E)$ were examined by pulse labeling using reporter constructs with consecutive AGR codons. Our results demonstrate that the $\mathrm{xm}^{5} \mathrm{U}$ modification plays a critical role in decoding NNG codons by stabilizing $U \cdot G$ pairing at the wobble position. Crystal structures of an anticodon stem-loop containing $\tau \mathrm{m}^{5} \mathrm{U}$ interacting with a UUA or UUG codon at the ribosomal A-site revealed that the $\tau \mathrm{m}^{5} \mathrm{U} \cdot \mathrm{G}$ base pair does not have classical $U \cdot G$ wobble geometry. These structures

\footnotetext{
* This work was supported by grants-in-aid from the Ministry of Culture, Sports, Science, and Technology of Japan (to T. S., T. O., and K. W.), by a Japan Society for the Promotion of Science Fellowship for Japanese Junior Scientists (to S. K.), by a grant from the New Energy and Industrial Technology Development Organization (to T.S.), by grants from the Medical Research Council (United Kingdom) and the Agouron Institute (to V. R.), and by a fellowship from the Austrian Academy of Sciences (to A. W.). The costs of publication of this article were defrayed in part by the payment of page charges. This article must therefore be hereby marked "advertisement" in accordance with 18 U.S.C. Section 1734 solely to indicate this fact.

The atomic coordinates and structure factors (code 2VQE and 2VQF) have been deposited in the Protein Data Bank, Research Collaboratory for Structural Bioinformatics, Rutgers University, New Brunswick, NJ (http://www.rcsb.org/).

5 The on-line version of this article (available at http://www.jbc.org) contains supplemental Figs. S1 and S2.

${ }^{1}$ To whom correspondence should be addressed. Tel.: 81-3-5841-8752; Fax: 81-3-3816-0106; E-mail: ts@chembio.t.u-tokyo.ac.jp.
}

provide help to explain how the $\tau \mathrm{m}^{5} \mathrm{U}$ modification enables efficient decoding of UUG codons.

The genetic code is deciphered by the anticodons of tRNAs, which carry an amino acid at the 3 ' end, bind to a specific codon in the mRNA, and transfer their amino acid to the growing polypeptide chain on the ribosome. In codon-anticodon interactions in the ribosome, the second and third bases (positions 35 and 36) of the anticodon base pair with the second and first bases of the codon, respectively, following Watson-Crick $(\mathrm{WC})^{2}$-type pairing rules. Structural studies of the $30 \mathrm{~S}$ ribosomal subunit revealed that the conserved bases A1492, A1493, and G530 in the decoding center of the $16 \mathrm{~S}$ rRNA specifically monitor these two WC-type pairings by A-minor interactions $(1,2)$. These interactions induce a large conformational rearrangement of the $30 \mathrm{~S}$ subunit that is necessary for tRNA selection and maintaining decoding fidelity. In contrast, base-pairing between the first base of the anticodon (position 34) and the third base of the codon does not always conform to WC-type pairing rules, so that synonymous codons for an amino acid are deciphered by a minimum set of tRNA anticodons. This behavior is referred to as "wobble pairing" (3), which is a highly evolved system required for the degeneracy of the genetic code by which 61 sense codons are deciphered into 20 amino acids by a limited set of tRNA species. Modified nucleosides are often found at the wobble position of tRNA anticodons $(4-8)$. The wobble modifications play critical roles in modulating codon recognition by restricting, expanding, or altering the decoding properties of the tRNAs (7). In contrast to the first and second codon-anticodon base pair, the ribosome imposes less restraints on the wobble base pair (1), so that various wobble

\footnotetext{
${ }^{2}$ The abbreviations used are: WC, Watson-Crick; MELAS, mitochondrial myopathy, encephalopathy, lactic acidosis, and stroke-like episodes; $\mathrm{Cmnm}^{5} \mathrm{U}, 5$-carboxymethylaminomethyluridine; $\pi \mathrm{m}^{5} \mathrm{U}, 5$-taurinomethyluridine; $\mathrm{mt}$, mitochondrial; DTT, dithiothreitol; MES, 4-morpholineethanesulfonic acid; ASL, anticodon stem loop; $\mathrm{xm}^{5} \mathrm{U}, 5$-methyluridine derivative; $\mathrm{xm}^{5} \mathrm{Um}, 5$-methyl-2' O-methyl derivative; $\mathrm{mnm}^{5} \mathrm{U}, 5$-methylaminomethyluridine; $\mathrm{mnm}^{5} \mathrm{~s}^{2} \mathrm{U}$, 5-methylaminomethyl-2-thiouridine; $\mathrm{mcm}^{5} \mathrm{U}, 5$-methoxycarbonylmethyluridine; $\mathrm{mcm}^{5} \mathrm{Um}, 5$-methoxycarbonylmethyl-2'-O-methyluridine.
} 
base pair geometries as well as modifications can readily be accommodated in the decoding center.

According to the original wobble rule (3), unmodified uridine at the wobble position (U34) was proposed to recognize only $\mathrm{A}$ and $\mathrm{G}$ at the third codon position. However, U34 can actually base-pair with any of the four bases due to its conformational flexibility (four-way wobbling). In fact, U34 is frequently found in tRNA species that are responsible for entire codon family boxes, in which four codons are synonymous, from Mycoplasma spp. and mitochondria (9-12). In bacteria and eukaryotic cytoplasm, uridines at the wobble position of tRNAs are often post-transcriptionally modified $(4,7)$. These modifications are classified into two groups according to their distinct chemical structures and decoding properties: 5-hydroxyuridine derivatives $\left(\mathrm{xO}^{5} \mathrm{U}\right)$ with an oxygen atom directly bonded to the $\mathrm{C} 5$ atom of the uracil base and 5-methyluridine derivatives $\left(\mathrm{xm}^{5} \mathrm{U}\right)$ with a methylene carbon directly bonded to the $\mathrm{C} 5$ atom. The $\mathrm{xo}^{5} \mathrm{U}$ type modifications are often found in bacterial tRNAs that are responsible for family boxes. Genetic and biochemical studies revealed that, in E. coli tRNAs, 5-carboxymethoxyuridine $\left(\mathrm{cmo}^{5} \mathrm{U}\right)$ is required to $\operatorname{read} \mathrm{A}, \mathrm{G}$, and $\mathrm{U}$ efficiently in vitro $(13,14)$ and to recognize all four bases in vivo in a mutant strain lacking other isoacceptors $(15,16)$. In contrast, $\mathrm{xm}^{5} \mathrm{U}$ type modifications are found at the wobble position of tRNAs that are responsible for purine-ending split codon boxes (NNR) (7). The $\mathrm{xm}^{5} \mathrm{U}$ type modifications include 2 -thiouridine derivatives $\left(\mathrm{xm}^{5} \mathrm{~s}^{2} \mathrm{U}\right)$ and $2^{\prime}$-O-methyluridine derivatives $\left(\mathrm{xm}^{5} \mathrm{Um}\right) .5$-Methylaminomethyluridine $\left(\mathrm{mnm}^{5} \mathrm{U}\right)$ and its 2-thio derivative $\left(\mathrm{mnm}^{5} \mathrm{~s}^{2} \mathrm{U}\right)$ are typical $\mathrm{xm}^{5} \mathrm{U}$ type modifications found in bacterial tRNAs, whereas 5-methoxycarbonylmethyluridine $\left(\mathrm{mcm}^{5} \mathrm{U}\right)$ and its 2 -thio $\left(\mathrm{mcm}^{5} \mathrm{~s}^{2} \mathrm{U}\right)$ and $2^{\prime}-O$ methyl derivatives $\left(\mathrm{mcm}^{5} \mathrm{Um}\right)$ are found only in eukaryotic tRNAs. 5-Carboxymethylaminomethyluridine $\left(\mathrm{cmnm}^{5} \mathrm{U}\right)$ (see Fig. 1A) can be found at the wobble position of tRNAs from Mycoplasma spp. and yeast mitochondria $(17,18)$. In bacteria, $\mathrm{cmnm}^{5}\left(\mathrm{~s}^{2}\right) \mathrm{U}$ is a modification intermediate of $\mathrm{mnm}^{5}\left(\mathrm{~s}^{2}\right) \mathrm{U}$, but $\mathrm{cmnm}^{5} \mathrm{~s}^{2} \mathrm{U}$ and $\mathrm{cmnm}^{5} \mathrm{Um}$ are also found at the wobble positions of $E$. coli tRNA ${ }^{\text {Leu4 } 4}$ and tRNA ${ }^{\text {Gln1 } 1}$, respectively. ${ }^{3}$ The conformation of $\mathrm{xm}^{5} \mathrm{~s}^{2} \mathrm{U}$ is largely fixed in the C3'-endo form of ribose puckering due to the large van der Waals radius of the 2 -thio atom causing steric repulsion of the $2^{\prime}$-oxygen atom (19). Due to its conformational rigidity, the $\mathrm{xm}^{5} \mathrm{~s}^{2} \mathrm{U}$ modification prefers to base-pair with A and prevents misreading of NNY codons $(6,19)$, regardless of the chemical characteristics of the C5-substituents. Compared with the 2-thio group of $\mathrm{xm}^{5} \mathrm{~s}^{2} \mathrm{U}$, the chemical nature and functional roles of C5-substituents of $\mathrm{xm}^{5} \mathrm{U}$ remain elusive.

The mammalian mitochondrial decoding system utilizes a limited set of tRNAs (22 species) that are capable of deciphering the 60 sense codons in the 13 protein genes encoded in mitochondrial (mt) DNA $(10,20)$. The wobble modifications play an essential role in this decoding system. The four-way wobble rule of $U 34$ reduces the total number of tRNA species required. In fact, each family box of codons is deciphered by a single tRNA with an unmodified wobble uridine. In human (and

${ }^{3} \mathrm{~K}$. Miyauchi and T. Suzuki unpublished result. bovine) mt tRNAs responsible for decoding purine-ending two-codon sets, we previously identified two novel $\mathrm{xm}^{5} \mathrm{U}$ wobble modifications that possess a sulfonic acid group derived from taurine: 5-taurinomethyluridine $\left(\tau \mathrm{m}^{5} \mathrm{U}\right)$ (see Fig. $\left.1 A\right)$ in tRNAs for Leu(UUR) and Trp and 5-taurinomethyl-2-thiouridine $\left(\tau \mathrm{m}^{5} \mathrm{~s}^{2} \mathrm{U}\right)$ in tRNAs for Lys, Gln, and Glu (21). ${ }^{4}$ These taurine-containing uridines are synthesized by direct incorporation of dietary taurine, indicating that taurine is a constituent of biological macromolecules and that there is a catabolic flow of intracellular taurine into mitochondria. Previously, we reported that the $\tau \mathrm{m}^{5}\left(\mathrm{~s}^{2}\right) \mathrm{U}$ modifications are defective in mutant tRNAs from cells harboring mitochondrial encephalomyopathies (22-25). The mutant A8344G mt tRNA ${ }^{\text {Lys }}$ from MERRF (myoclonus epilepsy associated with ragged red fibers) patients possesses an unmodified wobble uridine instead of the normal $\tau \mathrm{m}^{5} \mathrm{~s}^{2} \mathrm{U}$ modification $(23,26)$. In one of five pathogenic mutations associated with MELAS, a mutant mt tRNA ${ }^{\text {Leu(UUR) }}$ also lacks the normal $\tau \mathrm{m}^{5} \mathrm{U}$ modification $(22,24)$. Biochemical studies using an in vitro mitochondrial translation system revealed that the wild type tRNA ${ }^{\text {Leu(UUR) }}$ whose $\tau \mathrm{m}^{5} \mathrm{U}$ modification was surgically replaced by an unmodified uridine exhibited severely reduced UUG decoding but no decrease in UUA decoding (27). This finding strongly suggests that a UUG codon-specific translational defect of mutant mt tRNA ${ }^{\text {Leu(UUR) }}$ lacking the wobble modification is the primary cause of MELAS at the molecular level.

In this study, we examined the decoding properties of $\mathrm{cmnm}^{5} \mathrm{U}$ - and $\tau \mathrm{m}^{5} \mathrm{U}$-modified tRNAs using an E. coli in vitro translation system to elucidate the functional roles played by $\mathrm{xm}^{5} \mathrm{U}$ modifications. We chose to compare the $\mathrm{cmnm}^{5} \mathrm{U}$ modification with $\tau \mathrm{m}^{5} \mathrm{U}$ because $\mathrm{cmnm}^{5} \mathrm{U}$ contains glycine-derived substituents, the chemical characteristics of which are similar to the taurine-derived substituent of $\pi \mathrm{m}^{5} \mathrm{U}$. The decoding properties of the $\mathrm{xm}^{5} \mathrm{U}$ modification were also investigated in cells of the knock-out strain for the modification enzyme $m n m E$, which generates the $\mathrm{xm}^{5} \mathrm{U}$ modification. Finally, we directly observed by crystallography the nature of the $\tau \mathrm{m}^{5} \mathrm{U} \cdot \mathrm{G}$ base pair at the wobble position at the ribosomal A-site. Our results demonstrate the critical role played by $\mathrm{xm}^{5} \mathrm{U}$ modifications in decoding NNG codons.

\section{EXPERIMENTAL PROCEDURES}

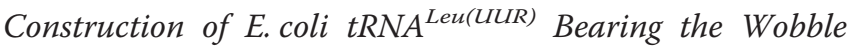
Modification-The $\mathrm{cmnm}^{5} \mathrm{U}$ and $\tau \mathrm{m}^{5} \mathrm{U}$ nucleosides were chemically synthesized $(21,28,29)$, and $5^{\prime}$ - and $3^{\prime}\left(2^{\prime}\right)$-diphosphorylation of these nucleosides was performed as previously described (30). The protocol for tRNA construction by molecular surgery is outlined in Fig. $1 C$ and was carried out as described previously (31-33), with slight modifications. pcmnm ${ }^{5} \mathrm{Up}$ or $\mathrm{p} \tau \mathrm{m}^{5} \mathrm{Up}$ was ligated to the $3^{\prime}$-end of the $5^{\prime}$-fragment (5'-HO-GGCCGGAUGGUGGAAUCGGUAGACACAAGGGAUU-OH-3') at $11^{\circ} \mathrm{C}$ for $16 \mathrm{~h}$ in a reaction mixture containing $50 \mathrm{~mm}$ Tris- $\mathrm{HCl}$ (pH 7.6), $15 \mathrm{~mm} \mathrm{MgCl}_{2}, 3.5 \mathrm{~mm}$ DTT, $15 \mu \mathrm{g} / \mathrm{ml}$ bovine serum albumin, $5 \%$ polyethylene glycol, $300 \mu \mathrm{M}$ ATP, $1.8 \mathrm{~mm}$ pcmnm ${ }^{5} \mathrm{Up}$ or $\mathrm{p} \tau \mathrm{m}^{5} \mathrm{Up}, 90 \mu \mathrm{M} 5^{\prime}$-fragment, and 1.6 units/ $\mu$ l T4 RNA ligase. Subsequently, periodate

${ }^{4}$ T. Suzuki and T. Suzuki, unpublished results. 
oxidation was performed to inactivate the unligated substrate (31). Sodium periodate $\left(\mathrm{NaIO}_{4}\right)$ was added to the reaction mixture to a final concentration of $10 \mathrm{~mm}$, and the solution was incubated on ice for $40 \mathrm{~min}$ in the dark. Sodium periodate was removed from the RNAs by ethanol precipitation. The 3'-phosphate of the ligated $5^{\prime}$-fragment was then removed using bacterial alkaline phosphatase, as described (32). The ligated 5 '-fragment and the 3 '-fragment (5'-HO-AAAAUCCCUCGGCGUUCGCGCUGUGCGGGUUCAAGUCCCGCUCCGGCUACCA-OH-3') (90 $\mu \mathrm{m}$ each) were mixed and heated at $65^{\circ} \mathrm{C}$ for $7 \mathrm{~min}$, annealed at room temperature for $1 \mathrm{~h}$ in $50 \mathrm{~mm}$ Tris- $\mathrm{HCl}$ (pH 7.6) and $15 \mathrm{mM} \mathrm{MgCl}_{2}$, and then ligated at $37^{\circ} \mathrm{C}$ for $1 \mathrm{~h}$ in buffer containing $60 \mathrm{~mm}$ Tris- $\mathrm{HCl}$ (pH 7.6), $17.5 \mathrm{~mm}$ $\mathrm{MgCl}_{2}, 3.5 \mathrm{~mm}$ DTT, $10 \mu \mathrm{g} / \mathrm{ml}$ bovine serum albumin, $300 \mu \mathrm{M}$ ATP, and 1.6 units/ $\mu$ l T4 RNA ligase. Subsequently, the $5^{\prime}$ termini of the tRNAs were phosphorylated (31). The resultant tRNAs were purified by separation on $10 \%$ denaturing PAGE. The sequences of the tRNAs were confirmed enzymatically (34) and by mass spectrometry fragment analysis (35-37) (supplemental Fig. S1). E. coli tRNA ${ }^{\text {Leu(UUR) }}$ having an unmodified wobble uridine was transcribed and purified as described (31).

Ribosomal A-site tRNA Binding-The A-site tRNA binding assay was carried out according to previously described methods $(2,27,38)$ with slight modifications. Briefly, the $5^{\prime}$-ends of E. coli tRNAs ${ }^{\mathrm{Leu}(\mathrm{UUR})}$ with or without wobble modifications were labeled with $\left[{ }^{32} \mathrm{P}\right]$ phosphate and mixed with unlabeled tRNA to adjust the concentration of each tRNA. mRNAs containing A-site UUR codons were synthesized by in vitro run-off transcription using T7 RNA polymerase as described (27). E. coli $70 \mathrm{~S}$ ribosomes were prepared as described (39). E. coli tRNA $^{\text {fMet }}$ was kindly provided by Dr. Nono Takeuchi (University of Tokyo). The ribosomal P-site was first occupied with initiator tRNA ${ }^{\text {fMet }}$ in a mixture $(10 \mu \mathrm{l})$ consisting of $5.2 \mathrm{pmol}$ of E. coli $70 \mathrm{~S}$ ribosome, $2 \mu \mathrm{g}$ of mRNA, 12.4 pmol of E. coli initiator tRNA ${ }^{\mathrm{fMet}}, 50 \mathrm{~mm}$ Tris- $\mathrm{HCl}$ (pH 7.5), $6.5 \mathrm{mM} \mathrm{MgCl}_{2}, 60 \mathrm{~mm}$ $\mathrm{KCl}, 1 \mathrm{~mm}$ DTT, and $2 \mathrm{~mm}$ spermine, which was incubated at $37^{\circ} \mathrm{C}$ for $17 \mathrm{~min}$. Four different amounts $(0.25,0.5,0.75$, and 1 pmol) of $5^{\prime}-^{32}$ P-labeled E. coli tRNAs ${ }^{\text {Leu(UUR) }}$ with or without wobble modifications in a mixture $(10 \mu \mathrm{l})$ consisting of $50 \mathrm{~mm}$ Tris- $\mathrm{HCl}$ (pH 7.5), $6.5 \mathrm{~mm} \mathrm{MgCl}_{2}, 60 \mathrm{~mm} \mathrm{KCl,} 1 \mathrm{~mm} \mathrm{DTT}$, and $2 \mathrm{~mm}$ spermine were added to the ribosomal mixtures, and a nonenzymatic binding reaction was performed at $37{ }^{\circ} \mathrm{C}$ for 12 $\mathrm{min}$. The reaction mixture was passed through nitrocellulose filters (pore size $0.45 \mu \mathrm{m}$; ADVANTEC). The filter was washed with $5 \mathrm{ml}$ of ice-cold buffer consisting of $50 \mathrm{~mm}$ Tris- $\mathrm{HCl}(\mathrm{pH}$ 7.5), $6.5 \mathrm{mM} \mathrm{MgCl}_{2}, 60 \mathrm{~mm} \mathrm{KCl}$, and $1 \mathrm{~mm}$ DTT and then airdried. The amount of tRNA bound was measured by liquid scintillation counting.

In Vitro Cell-free Translation-The in vitro cell-free translation assay was carried out according to previously described methods. Briefly, tRNAs ${ }^{\text {Leu(UUR) }}$ with or without wobble modifications were leucylated at $37^{\circ} \mathrm{C}$ for $10 \mathrm{~min}$ in a reaction mixture $(30 \mu \mathrm{l})$ consisting of $100 \mathrm{~mm}$ Tris- $\mathrm{HCl}(\mathrm{pH}$ 7.6), $5 \mathrm{~mm}$ $\mathrm{MgCl}_{2}, 2 \mathrm{~mm}$ ATP, $20 \mathrm{~mm} \mathrm{KCl}, 1 \mathrm{~mm}$ DTT, 20\% dimethyl sulfoxide, $100 \mu \mathrm{M}\left[{ }^{14} \mathrm{C}\right] \mathrm{L}-$ leucine, and $1 \mu \mathrm{g} / \mu \mathrm{l}$ E. coli leucyl-tRNA synthetase. The preparation of E. coli S30 extracts has been described previously (40). Four UUN-mRNAs, each containing one UUN test codon, were synthesized in vitro using T7 RNA polymerase, as described (41), to create the following open reading frame sequence (test codon underlined): 5 '-AUGAUCAUUAUCAUUAUCAUUAUCAUAAUCAUCUUNGUGGUGGUCGUGGUGUAAUAGUAG-3', which encodes Met$\mathrm{Ile}_{10}-\mathrm{Leu} / \mathrm{Phe}^{-V_{\mathrm{Val}}}{ }_{5}$. To construct template DNAs for UUN-mRNAs, DNA fragments were synthesized by Klenow reaction using the following primers: 5'-GAAGGAGATATACATATGATCATTATCATTATCATTATCATAATCATCTTNGTGGTGGTCGTGGTGTA-3' and 5'-GACACAGGAAACAGCTATGACCATGATTACGCCAAGCTTATGCATCTACTATTACACCACGACCACCAC-3'. The template DNAs were then subjected to PCR to obtain the insert fragments for the templates using the following primers: $5^{\prime}$-CCGGGTAATACGACTCACTATAGGGAGACCACAACGGTTTCCCTCTAGAAATAATTTTGTTTAACTTTAAGAAGGAGATATACATATGATC-3' and 5' -AAAAAAAAAACGAGCCTTTCGGCTCGTATGTTGTGTGGAATTGTGAGCGGATAACAATTTCACACAGGAAACAGCTATG-3'. Resultant DNAs were inserted into the BamHI/EcoRI site of pUC19 (TOYOBO). The nucleotide sequences of the plasmids encoding UUN-mRNAs were confirmed by the dideoxy termination method of sequencing using a 3100 Genetic Analyzer (Applied Biosystems). Each template DNA was prepared from large scale cultures of E. coli JM109 cells, completely digested with EcoRI, and then transcribed with T7 RNA polymerase. In addition, we prepared an mRNA (GGC-mRNA) in which the UUN codon was replaced with a GGC codon, to be used as a negative control. The cell-free translation reaction $(18.7 \mu \mathrm{l})$ contained 44 mM HEPES-KOH (pH 7.5), 11 mM DTT, 1.8 mM GTP, 8.4 mM phosphoenolpyruvate potassium salt, $1.5 \mathrm{~mm}$ ATP, $0.8 \%(\mathrm{w} / \mathrm{v})$ polyethylene glycol 8000 (Sigma), $0.54 \mathrm{mg} / \mathrm{ml}$ folinic acid calcium salt (Sigma), $44 \mathrm{~mm}$ ammonium acetate, $6.4 \mathrm{~mm}$ spermidine, $6 \mathrm{~mm}$ magnesium acetate, $56 \mathrm{~mm}$ potassium glutamate, 0.3 $\mathrm{mm}$ each of methionine, isoleucine, and valine, $1 \mu \mathrm{g}$ of one of the mRNAs, 5 pmol of $\left[{ }^{14} \mathrm{C}\right]$ Leu-tRNA ${ }^{\text {Leu(UUR) }}$ with or without wobble modification, and one-sixth volume of S30 extract. The mixture was incubated at $37^{\circ} \mathrm{C}$ for $15 \mathrm{~min}$, and the radioactivity of amino acids incorporated into the peptide was measured by liquid scintillation counting. Radioactivity of incorporated Leu into GGC-mRNA was subtracted from that of each UUN-mRNA to obtain decoding activity data.

Construction of E. coli $\triangle m n m E$ Strain-The E. coli K-12 strain BW25113 (lacl ${ }^{\mathrm{q}} \mathrm{rrnB}_{\mathrm{T} 14} \Delta$ lac $_{\mathrm{W} 116}$ hsdR514 $\Delta$ araBA$\left.D_{\mathrm{AH} 33} \Delta r h a B A D_{\mathrm{LD} 78}\right)$ was used for the "one-step inactivation of chromosomal genes" procedure (42). Briefly, a PCR fragment containing the kanamaycin resistance $(\mathrm{kan})$ gene flanked by two flippase recombinase (FLP) recognition targets was generated using $\mathrm{pKD} 4$ as the template with the following primers: KO-F, 5' -TAAGCACCGCGCATCCGCCACACAAAGCAACAGGAACATCGTGTAGGCTGGAGCTGCTTC-3'; KO-R, 5' -AGCCGCATCTGACAGTCAGAATGCGGCTTCGTAAGCGCGGCATATGAATATCCTCCTTAGT-3'. This PCR product contains 40 nucleotide extensions that are homologous to the $3^{\prime}$ and 5' 40 base pairs of $m n m E$. The PCR fragment was introduced into BW25113/pKD46 E.coli, resulting in insertion of the kan FLP recognition target cassette into the $m n m E$ gene. Disruption of the $m n m E$ gene was confirmed by PCR using $5^{\prime}$-TACATGCTGATGGGTTCCGT- ${ }^{\prime}$ and 
5'-GAGGTCACACATATATGTAA-3' as the primers. The $m n m E$ disruption was transduced into BW25113, and the resistance gene was removed using an FLP expression plasmid (pCP20).

Construction of fusA Reporters-The E. coli fusA gene encoding EF-G was cloned into the BamHI/SalI site of pQE-80L (Qiagen). The codons in the fusA gene corresponding to Val-447, Trp-448, and Thr- 449 were mutated to AGR codons using a QuikChange mutagenesis kit (Stratagene) according to the manufacturer's instruction to create the fus $A$ reporters using the following primers: $5^{\prime}$-AAGACCCGTCTTTCCGTAGAAGAAGAGACGAAGAATCTAACCAG-3' and 5' -CTGGTTAGATTCTTCGTCTCTTCTTCTACGGAAAGACGGGTCTT-3' for fusA-AGA; 5' ACCCGTCTTTCCGTGTAAGGAGGGACGAAGAATCTAACCAG-3' and 5'-CTGGTTAGATTCTTCGTCCCTCCTTACACGGAAAGACGGGT-3' for fusA-AGG. Construction of the reporters was confirmed by DNA sequencing using a 3100 Genetic Analyzer (Applied Biosystems). The pArgU plasmid, a pMW218 harboring the E. coli $\arg U$ gene, was kindly provided by Dr. Yamada (Mitsubishi Chemical Corp.).

Pulse-labeling of Nascent Peptide Chains-Pulse labeling with $\left[{ }^{35} \mathrm{~S}\right]$ methionine was performed as described previously (43) with slight modifications. Each fusA reporter (fusA-AGA and fusA-AGG) was introduced into an E. coli wild-type or $\Delta m n m E$ strain. The cells were grown at $37^{\circ} \mathrm{C}$ to OD 0.4 at $A_{600}$ in LB medium ( $2 \mathrm{ml}$ ), and isopropyl 1-thio- $\beta$-D-galactopyranoside was then added to the medium to a final concentration of 1 $\mathrm{mm}$ for induction. After a $30-\mathrm{min}$ induction, $\left[{ }^{35} \mathrm{~S}\right]$ methionine (final concentration, $110 \mu \mathrm{Ci} / \mathrm{ml}$ ) was added to start the pulse labeling $(t=0)$. At $20 \mathrm{~s}$, unlabeled methionine (final concentration, $20 \mathrm{~mm}$ ) was rapidly mixed into the medium. Samples (100- $\mu$ l aliquots) were taken at $t=10,20,30,40,60,80,110$, 150 , and $200 \mathrm{~s}$ and transferred into new tubes containing liquid nitrogen. Before thawing, chloramphenicol was added to a final concentration of $200 \mu \mathrm{g} / \mathrm{ml}$. The cells were washed twice at $4{ }^{\circ} \mathrm{C}$ with double-distilled $\mathrm{H}_{2} \mathrm{O}$. Washed pellets were suspended in a mixture $(6.5 \mu \mathrm{l})$ consisting of $50 \mathrm{mM}$ HEPES-KOH ( $\mathrm{pH} 7.6)$, $100 \mathrm{~mm} \mathrm{KCl}, 10 \mathrm{~mm} \mathrm{MgCl}_{2}$, and $0.2 \mathrm{~mm}$ phenylmethylsulfonyl fluoride. To this suspension, $2.5 \mu \mathrm{l}$ of sample buffer $(250 \mathrm{~mm}$ Tris- $\mathrm{HCl}$ (pH 6.8), 40\% glycerol, $8 \%$ SDS, and $0.005 \%$ bromophenol blue) and $1 \mu \mathrm{l}$ of 2-mercaptoethanol were added, and the samples were then boiled at $95^{\circ} \mathrm{C}$ for $5 \mathrm{~min}$. The lysates were analyzed by SDS-PAGE on a $10-20 \%$ polyacrylamide gradient gel (Wako Chemicals). The gel was stained with Coomassie Brilliant Blue R250, washed, and vacuum-dried. The gel was exposed to an imaging plate, and the labeled polypeptides were visualized using a bioimaging analyzer (BAS 5000; Fuji Photo Film).

Northern Blots-Total RNA from E. coli was isolated using ISOGEN (Nippon Gene), according to the manufacturer's instructions. The total RNA $(\sim 5 \mu \mathrm{g})$ was electrophoresed on a $10 \%$ denaturing polyacrylamide gel and blotted onto a nylon Hybond $\mathrm{N}$ membrane (Amersham Biosciences) with $1 \times \mathrm{TBE}$ using a Transblot SD apparatus (Bio-Rad). The membrane was air-dried, and the blotted RNA was fixed onto the membrane by UV irradiation $\left(254 \mathrm{~nm}, 360 \mathrm{~mJ} / \mathrm{cm}^{2}\right)$. Northern blotting was conducted using a standard protocol (44). Oligonucleotide probes (5' -CCTGCGGCCCACGAC-3' for tRNA ${ }^{\text {Arg-4 } 4}$ and 5' -
AACCTGCAATTAGCCC-3' for tRNA ${ }^{\text {Arg-5}}{ }^{\prime}$ ) were $5^{\prime}$-end-labeled with ${ }^{32} \mathrm{P}$. Radioactivity was visualized by exposing the membrane to an imaging plate and analyzing with a bioimaging analyzer (BAS 5000; Fuji Photo Film).

Construction of Anticodon Stem Loop (ASL) Bearing $\tau m^{5} U-$ The procedure to construct ASL containing $\tau \mathrm{m}^{5} \mathrm{U}$ by molecular surgery was basically carried out as described previously (3133). $\mathrm{p} \tau \mathrm{m}^{5} \mathrm{Up}$ was ligated with the $3^{\prime}$-end of the $5^{\prime}$-fragment ( $5^{\prime}$-HO-GCAUGCU-OH- $\left.3^{\prime}\right)$ at $11^{\circ} \mathrm{C}$ for $16 \mathrm{~h}$ in the reaction mixture containing $50 \mathrm{~mm}$ Tris- $\mathrm{HCl}(\mathrm{pH} 7.6), 15 \mathrm{~mm}$ magnesium chloride, $3.5 \mathrm{~mm} \mathrm{DTT}, 15 \mu \mathrm{g} / \mathrm{ml}$ bovine serum albumin, $5 \%$ polyethylene glycol, $300 \mu \mathrm{M}$ ATP, 10\% DMSO, $1.8 \mathrm{~mm}$ $\mathrm{p} \tau \mathrm{m}^{5} \mathrm{Up}, 90 \mu \mathrm{m} 5^{\prime}$-fragment (5'HO-GCAUGCU-OH-3'), and 1.6 units/ $\mu \mathrm{l}$ T4 RNA ligase. Ligation efficiency was $35 \%$. The ligated 5 '-fragment was separated from unligated ones by anion exchange chromatography using a MonoQ HR 5/5 column (GE Healthcare) followed by desalting using Sep-Pak plus C18 Cartridges (Waters). The 3 '-phosphate of the ligated 5 '-fragment was removed using bacterial alkaline phosphatase. The 3 '-fragment (5'-HO-AAAACAUGCU-OH-3') was 5' -phosphorylated using $\mathrm{T} 4$ polynucleotide kinase, and then the $3^{\prime}$ terminal residue was removed by periodate oxidation to obtain $5^{\prime}-\mathrm{p}$ AAAACAUGC-p- $3^{\prime}$. The $5^{\prime}$ - and $3^{\prime}$-fragments were then ligated at $11^{\circ} \mathrm{C}$ for $16 \mathrm{~h}$ in the reaction mixture containing $50 \mathrm{~mm}$ Tris- $\mathrm{HCl}$ (pH 7.6), $15 \mathrm{~mm}$ magnesium chloride, $3.5 \mathrm{~mm}$ DTT, 15 $\mu \mathrm{g} / \mathrm{ml}$ bovine serum albumin, $5 \%$ polyethylene glycol, $300 \mu \mathrm{M}$ ATP, 10\% DMSO, $90 \mu \mathrm{M} 5$ ' -fragment, $450 \mu \mathrm{M} 33^{\prime}$-fragment, and 1.6 units/ $\mu \mathrm{l}$ T4 RNA ligase. Ligation efficiency was $42 \%$. The resultant ASL was purified by MonoQ column chromatography. The sequence and chemical structure of the ASL was confirmed by mass spectrometry analysis as described $(35,36)$.

Crystallization, Data Collection, and Refinement of the $30 \mathrm{~S}$ Complexes-Thermus thermophilus $30 \mathrm{~S}$ ribosomal subunits were purified, crystallized, and cryoprotected as described (45) (the final solution being 26\% (v/v) MPD, $100 \mathrm{~mm}$ potassium$\operatorname{MES}$ (pH 6.5), $200 \mathrm{~mm} \mathrm{KCl,} 75 \mathrm{~mm} \mathrm{NH}_{4} \mathrm{Cl}$, and $15 \mathrm{mM} \mathrm{MgCl}_{2}$ ).

The mRNA oligonucleotides 5'-UU(A/G)AAA-3' were chemically synthesized (Dharmacon) and gel purified. After cryoprotection, the $30 \mathrm{~S}$ crystals were soaked in cryoprotection buffer containing $80 \mu \mathrm{M}$ paromomycin, $300 \mu \mathrm{M} \mathrm{ASL} \mathrm{L}_{\tau \mathrm{m} 5 \mathrm{UAA}}^{\mathrm{Leu}}$ and $300 \mu \mathrm{M}$ of the corresponding mRNA hexanucleotide for at least $48 \mathrm{~h}$ as described $(1,2)$. Crystals were flash-cooled in liquid nitrogen and stored for data collection. Crystals were prescreened at European Synchrotron Radiation Facility beamline ID14-2 using short exposures $90^{\circ}$ apart. Crystals were then stored in liquid nitrogen before data were collected at European Synchrotron Radiation Facility beamline ID14-4 and the Swiss Light Source beamline X10SA in a cryostream at 90-100 K. Processing was done using XDS (46). The CCP4 package was used for assorted tasks (47). Coot was used for visualization and building (48), and CNS 1.2 was used for refinement (49). Topologies and parameters were used directly or derived using HIC-Up (50). A summary of crystallographic data is shown in Table 1. Differences in the individual data sets are likely to be the result of slight differences in crystal quality. Figures as well as alignments between the individual structures were made using PyMOL (W. L. DeLano; available on the World Wide Web). The structure of an RNA A-form helix containing a G:U 
A
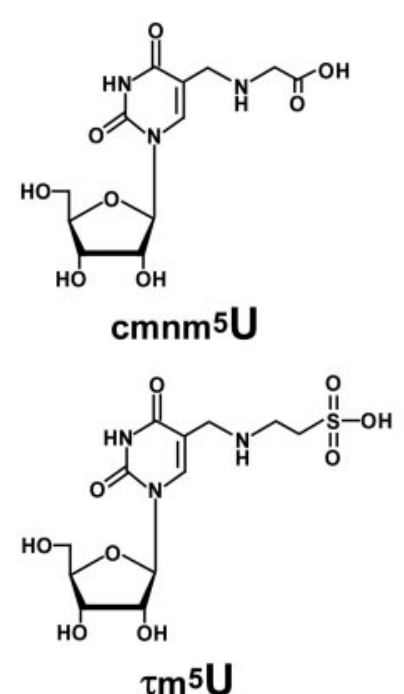

$\tau \mathrm{m}^{5} \mathrm{U}$

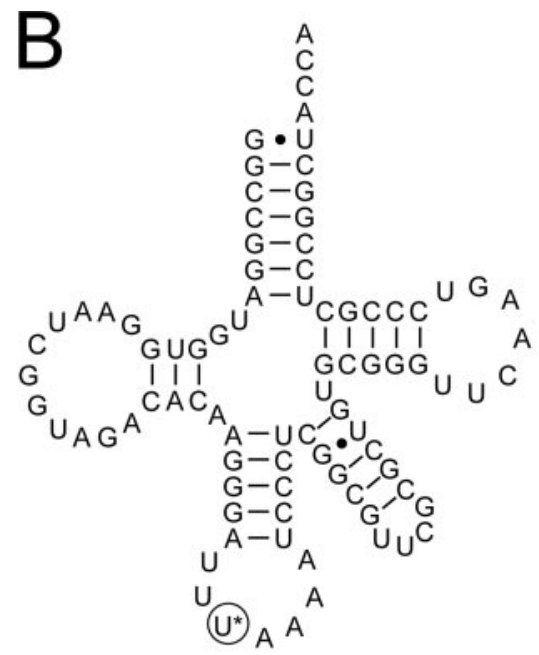

E.coli tRNA ${ }^{\mathrm{Leu}(U \mathrm{U})}$
C

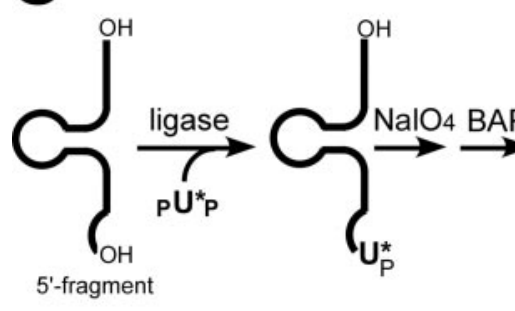

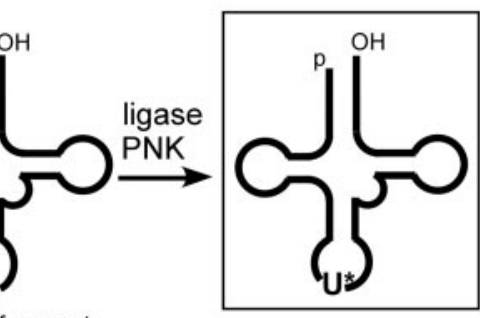

E. coli tRNA ${ }^{\text {Leu(UUR) }}$ with $U^{\star} 34$
FIGURE 1. Construction of $E$. coli tRNA ${ }^{\text {Leu(UUR) }}$ bearing wobble modifications. $A$, chemical structures of the modified uridines introduced into the wobble position of $E$. coli tRNA ${ }^{\text {Leu(UUR): }}: \mathrm{cmnm}^{5} U$ and $\tau \mathrm{m}^{5} U$. $B$, cloverleaf structure of $E$. coli tRNA ${ }^{\text {Leu(UUR) }}$. $U^{*}$, the modified uridine. $C$, schematic depiction of the molecular surgery procedure used to construct $E$. coli tRNA ${ }^{\text {Leu(UUR) }}$ bearing the wobble modifications. The details are described under "Experimental Procedures." BAP, bacterial alkaline phosphatase; $P N K$, polynucleotide kinase.

tion. Modified uridines $\left(\mathrm{U}^{*}\right)$ of $\mathrm{cmnm}^{5} \mathrm{U}$ and $\pi \mathrm{m}^{5} \mathrm{U}$ (Fig. $1 A$ ) were chemically synthesized, as described previously $(21,28,29)$, and the $5^{\prime}$ - and $3^{\prime}$ (or 2')-hydroxyl groups of each modified uridine were phosphorylated to obtain $\mathrm{pU}$ "p as a monomer unit for enzymatic ligation. The procedure for construction of tRNA variants is outlined in Fig. 1C. The $5^{\prime}$-fragment is ligated to $\mathrm{pU}$ "p using T4 RNA ligase. The efficiency of this reaction was estimated to be $\sim 50 \%$ for $\mathrm{pcmnm}^{5} \mathrm{Up}$ and $\sim 30 \%$ for $\mathrm{p} \pi \mathrm{m}^{5} \mathrm{Up}$. The efficiency of the second ligation, between $5^{\prime}$ and $3^{\prime}$ fragments, was greater than $80 \%$ in both cases. The final yield of each tRNA variant was $\sim 10 \%$ for tRNA bearing the $\mathrm{cmnm}^{5} \mathrm{U}(144 \mu \mathrm{g})$ and $\sim 5 \%$ for tRNA bearing the $\tau \mathrm{m}^{5} \mathrm{U}$ $(132 \mu \mathrm{g})$. E. coli tRNA ${ }^{\text {Leu(UUR) }}$ bearing an unmodified U34 was prepared by run-off transcription. The primary sequence for each modified tRNA was confirmed by enzymatic digestion (34) (data not shown) as well as by mass spectrometric analysis (supplemental Fig. S1). Each tRNA was efficiently aminoacylated by E.coli leucyl-tRNA synthetase with an acceptor activity of about $900 \mathrm{pmol} /$ $A_{260}$ unit.

Binding of the tRNA Variants to
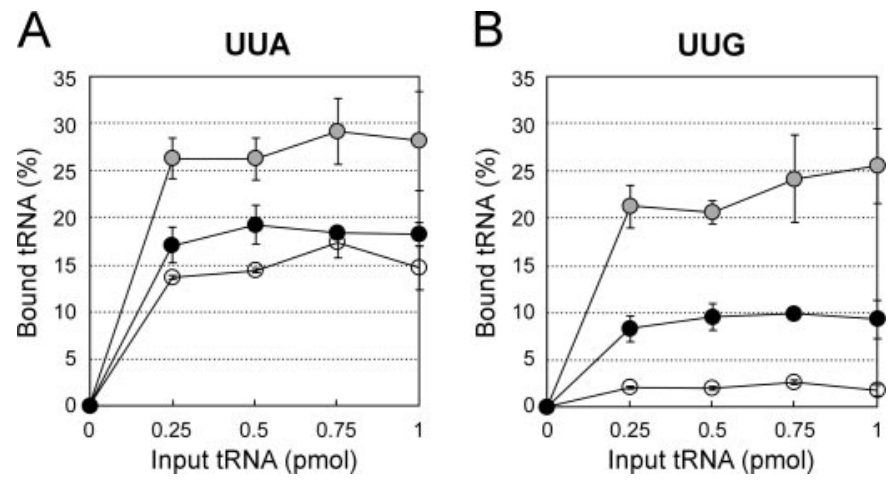

FIGURE 2. Ribosomal A-site tRNA binding of the UUA $(A)$ and UUG $(B)$ codons. Shown is E. colitRNA ${ }^{\text {Leu(UUR) }}$ bearing an unmodified wobble uridine (blank circle), $\mathrm{cmnm}^{5} \mathrm{U}$ (gray circle), and $\mathrm{m}^{5} \mathrm{U}$ (black circle). Three independent experiments were performed, and the average values are plotted. Bars, S.D.

wobble base pair was aligned to the codon-anticodon helix to compare it with the $\pi \mathrm{m}^{5} \mathrm{U} \cdot \mathrm{G}$ base pair (52).

\section{RESULTS}

Construction of E. coli $t R N A^{\text {Leu(UUR) }}$ with or without $x m^{5} U$ Modifications - To examine the decoding properties of $\mathrm{xm}^{5} \mathrm{U}$ modifications at the wobble position using an in vitro translation system, we constructed two E. coli tRNA ${ }^{\text {Leu(UUR) }}$ variants (Fig. 1B) in which $\mathrm{cmnm}^{5} \mathrm{U}$ and $\tau \mathrm{m}^{5} \mathrm{U}$ were introduced at the wobble posi-
UUR Codons at the Ribosomal A-site-Each tRNA variant was used in ribosomal A-site binding experiments to measure the efficiency of $\mathrm{xm}^{5} \mathrm{U}$ modifications in codon recognition. The strength of codon-anticodon pairing on the ribosome is quantified by tRNA binding to the ribosomal A-site occupied by a specific codon $(2,27)$. For these assays, we designed short mRNAs (49 nt) containing an AUG codon at the P-site and one of four UUN codons at the A-site, downstream of a standard Shine-Dalgarno sequence. Prior to the A-site tRNA binding, the P-site is occupied by tRNA ${ }^{\mathrm{fMet}}$. As shown in Fig. $2, A$ and $B$, the tRNA ${ }^{\text {Leu(UUR) }}$ with U34 showed efficient binding to the UUA codon (15\%) but weak binding to the UUG codon ( $2 \%$ ). In contrast, the tRNA ${ }^{\text {Leu(UUR) }}$ with $\mathrm{cmnm}^{5} \mathrm{U} 34$ exhibited high levels of binding to both codons ( $28 \%$ for UUA and $25 \%$ for UUG). The tRNA ${ }^{\text {Leu(UUR) }}$ with $\tau \mathrm{m}^{5} \mathrm{U} 34$ bound to both codons with relatively lower affinity ( $15 \%$ for UUA and $10 \%$ for UUG) than the $\mathrm{cmnm}^{5}$ U34-modified tRNA ${ }^{\mathrm{Leu}(U U R)}$. These results suggest that $\mathrm{xm}^{5} \mathrm{U}$ modifications are required to interact with the UUG codon at the ribosomal A-site.

Translational Activity of tRNA Variants Measured by E. coli Cell-free Translation-Codon-specific translational activity of tRNA variants, with or without an $\mathrm{xm}^{5} \mathrm{U} 34$ modification, was assessed using an in vitro E. coli translation system (40). Four mRNAs, each containing one of the UUN test codons at a single site, were synthesized. Using these constructs, we estimated the 


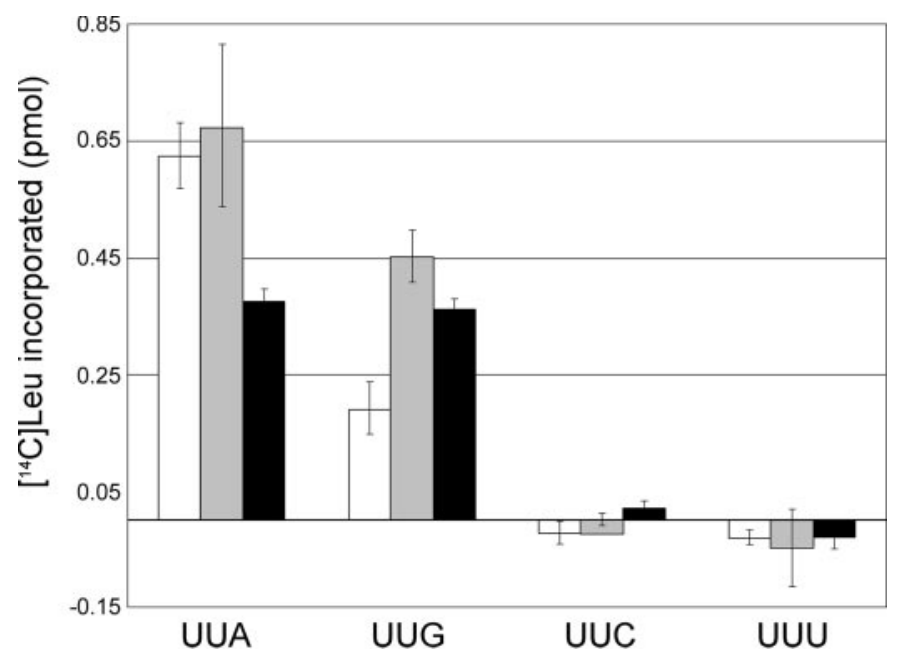

FIGURE 3. Translational activity of $E$. coli tRNA ${ }^{\text {Leu(UUR) }}$ containing $\mathbf{c m n m}^{5} \mathbf{U}$ or $\boldsymbol{\tau m}^{5} \mathbf{U}$. Translational activity of $E$. coli tRNA ${ }^{\text {Leu(UUR) }}$ bearing an unmodified wobble uridine (white bars), $\mathrm{cmnm}^{5} \mathrm{U}$ (gray bars), or $\tau \mathrm{m}^{5} \mathrm{U}$ (black bars) was quantified by measuring $\left[{ }^{14} \mathrm{C}\right]$ Leu incorporation for each mRNA. Data of GGC-mRNA as a negative control was subtracted from each data.

decoding activity of the tRNAs for each UUN codon at each round of in vitro translation. As shown in Fig. 3, each of the tRNA ${ }^{\text {Leu(UUR) }}$ variants specifically translated UUR codons but was unable to translate UUY codons, demonstrating that codon-specific translational activity can be measured by this system. According to the four-way wobble rule, the E. coli tRNA $^{\text {Leu(UUR) }}$ with U34 should translate UUY codons, but it was in fact completely unable to decode UUY codons. This result is consistent with our previous study assessing mitochondrial translation (27). The tRNA ${ }^{\text {Leu(UUR) }}$ with U34 efficiently translated the UUA codon, but decoding of the UUG codon was approximately one-third the activity of decoding UUA. As observed in Fig. $2 B$, the unmodified tRNA ${ }^{\text {Leu(UUR) }}$ showed little binding activity to the ribosomal A-site. However, in this experiment, EF-Tu enzymatically delivers leucyl-tRNAs to the A-site, which might stimulate the activity of the unmodified tRNA on UUG decoding. In contrast, the tRNA ${ }^{\text {Leu(UUR) }}$ with $\mathrm{cmnm}^{5} \mathrm{U} 34$ exhibited efficient translation of both UUR codons (Fig. 3). In fact, the decoding activity of the $\mathrm{cmnm}^{5}$-modified wobble uridine was not significantly different from UUA decoding by U34 tRNA ${ }^{\mathrm{Leu}(U \mathrm{UR})}$, but the $\mathrm{cmnm}^{5}$ modification stimulated twice as much decoding activity of the UUG codon as observed for the U34, indicating that the C5-substituent of $\mathrm{xm}^{5} \mathrm{U} 34$ is required for efficient translation of NNG codons. In the case of the tRNA ${ }^{\text {Leu(UUR) }}$ with $\tau \mathrm{m}^{5} \mathrm{U} 34$ (Fig. 3), the $\tau \mathrm{m}^{5}$ modification caused a reduction in UUA decoding, but the level of UUG decoding was increased almost 2-fold. Since $\pi \mathrm{m}^{5} \mathrm{U}$ is a mitochondrial wobble modification, the heterologous combination of translational machinery may result in the reduced activity of tRNA ${ }^{\mathrm{Leu}(\mathrm{UUR})}$ with $\tau \mathrm{m}^{5} \mathrm{U} 34$. Considering that the U34 tRNA ${ }^{\text {Leu(UUR) }}$ translated the UUA codon more efficiently than the UUG codon and that the $\tau \mathrm{m}^{5} \mathrm{U} 34$ translated both codons at similar levels, the $\tau \mathrm{m}^{5}$ modification plays a role in enhancing the decoding of UUG codons.

During the cell-free translation, some part of ${ }^{14} \mathrm{C}$-labeled leucyl-tRNA might be deacylated to produce free $\left[{ }^{14} \mathrm{C}\right]$ Leu with a submicromole level. Since S30 extract contains intrinsic
tRNA $^{\text {Leu }}$ for decoding UUR codons, it is possible that intrinsic tRNA $^{\text {Leu }}$ can be acylated with the labeled Leu, which might be incorporated into the UUR codons in the synthesized mRNAs. Considering the high $K_{m}$ value (few mM) of amino acid for aminoacyl-tRNA synthetases, it is not enough concentration for efficient aminoacylation. In addition, an excess amount of the adscititious tRNA ${ }^{\text {Leu }}$ may be dominantly reaminoacylated as compared with the intrinsic tRNA ${ }^{\text {Leu }}$ in the S30 extract. Although a trace amount of Leu incorporation into the UUR codons by the intrinsic tRNA ${ }^{\text {Leu }}$ still cannot be ruled out, it is not a critical issue to estimate the decoding activity in this method (40).

Pulse Labeling Experiments to Estimate the Decoding Activity of $t R N A^{\text {Arg }}$ with or without Wobble Modifications in Vivo-In E. coli, AGR are utilized as rare codons decoded by two minor tRNAs: tRNA ${ }^{\mathrm{Arg} 4}$ with the mnm ${ }^{5} \mathrm{UCU}$ anticodon, which is responsible for both AGA and AGG codons, and tRNA ${ }^{\mathrm{Arg} 5}$ with the CCU anticodon, which is responsible for the AGG codon alone (53). Ribosomes tend to stall at consecutive AGR codons, giving rise to truncated nascent peptides $(54,55)$. To examine the decoding activity of tRNA ${ }^{\text {Arg4 }}$ with or without an mnm ${ }^{5} U C U$ modification in the cell, we devised a pulse-chase radiolabeling experiment using two reporter constructs. E. coli fus $A$ was employed as a reporter gene in which consecutive AGR codons were artificially inserted in the middle of the coding sequence (Fig. 4A). We inserted three consecutive AGA codons (fusA-AGA) or two tandem AGG codons (fusA-AGG) in the reporter constructs, because the NNG codon is translated much more slowly than the NNA codon in two codon sets (56). For each construct, $\left[{ }^{35} \mathrm{~S}\right]$ methionine was added into the medium after induction of the fusA reporter and then incubated for $20 \mathrm{~s}$ to specifically label the nascent peptides of the fusA gene. The cultures were then chased with cold methionine to monitor the labeled peptides over time. In wild-type cells harboring the fusA-AGA construct (Fig. 4B), we clearly observed a distinct band of $\sim 52 \mathrm{kDa}$ corresponding to the nascent peptide of fusA stalled at the consecutive AGA codons, and only a minor band corresponding to the mature form of fus $A$ could be detected. $m n m E$ is the gene for $\mathrm{mnm}^{5} \mathrm{U}$ biogenesis, so that tRNA $^{\text {Arg4 }}$ contains unmodified U34 in the knock-out $M n m E$ strain $(\Delta m n m E)$ (57). In $\Delta m n m E$ cells harboring the fusA-AGA construct, we could see the same nascent fus $A$ peptide. This result suggested that the consecutive AGA codons were not efficiently decoded by tRNA ${ }^{\mathrm{Arg} 4}$ even when it bears a normal $\mathrm{mnm}^{5} \mathrm{U}$ modification. When a plasmid bearing the tRNA $^{\text {Arg4 }}$ gene (pArgU) was introduced, the radiolabeled nascent peptide disappeared, and the mature fus $A$ product clearly appeared in both wild-type and $\triangle m n m E$ strains (Fig. $4 B$ ). Thus, the consecutive AGA codons were efficiently translated by overexpressed tRNA ${ }^{\text {Arg4 }}$ even when it contained an unmodified wobble uridine. In contrast, with the fus A-AGG construct (Fig. $4 C$ ), mature fus $A$ was mainly observed in the wild-type strain. In the $\Delta m n m E$ strain, the nascent fus $A$ peptide was observed as a major product, whereas the mature fus $A$ was a minor product, which was presumably produced by the minor tRNA ${ }^{\text {Arg5 }}$ capable of decoding AGG codons. This result demonstrated that the $\mathrm{mnm}^{5} \mathrm{U}$ modification was required to decode tandem AGG codons efficiently. When pArgU was introduced in the $\Delta m n m E$ 
Wobble Modifications Stabilize U.G Pairing

A

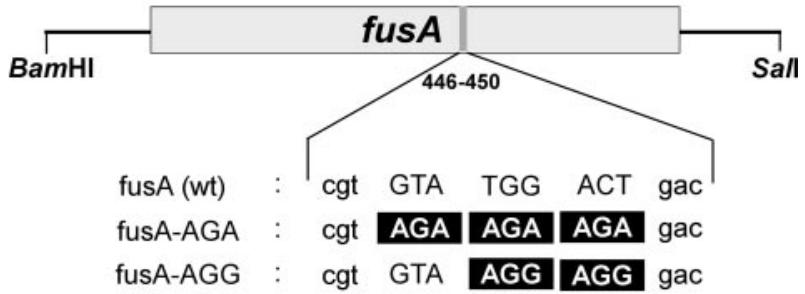

B

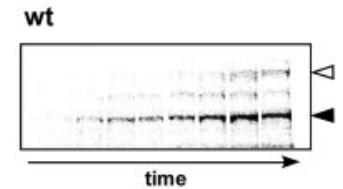

$\Delta m n m E$

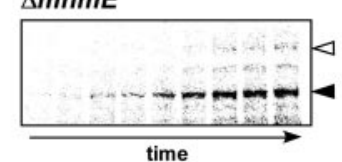

wt +pArgU

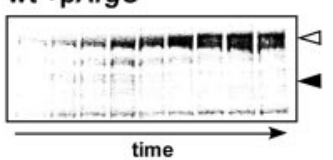

C

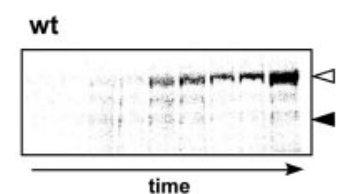

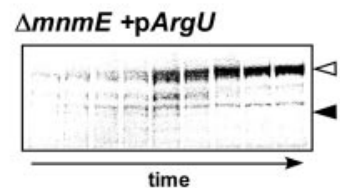

$\Delta m n m E$

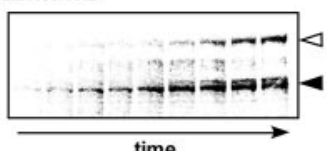

time

$\Delta m n m E+p A r g U$

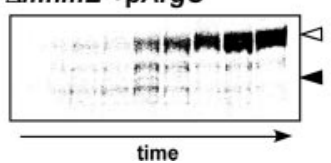

FIGURE 4. Pulse-labeling experiments to estimate the decoding activity of tRNA ${ }^{\mathrm{Arg}-4}$. $A$, reporter constructs. DNA sequences at codon positions 446450 of the fus $A$ gene are magnified to show the mutated sequence for each reporter. Shown are ${ }^{35} \mathrm{~S}$-radiolabeled bands of fusA-AGA $(B)$ and fusA-AGG $(C)$ in the wild-type (top left), wild-type harboring pAugU (bottom left), $\Delta m n m E$ (top right), and $\Delta m n m E$ harboring $\mathrm{pAugU}$ (bottom right) strains. Lanes correspond to $10,20,30,40,60,80,110,150$, and $200 \mathrm{~s}$ after initiation of the pulse labeling.

strain harboring the fus A-AGG construct, the nascent peptides disappeared, and mature products of fus $A$ increased, indicating that efficient decoding of tandem AGG codons by the rare tRNA ${ }^{\mathrm{Arg} 4}$ with the $\mathrm{mnm}^{5} \mathrm{UCU}$ anticodon can be compensated by tRNA ${ }^{\mathrm{Arg} 4}$ with a UCU anticodon if it is abundantly expressed in the cell. For each construct, steady-state levels of tRNA ${ }^{\text {Arg4 }}$ were measured by Northern blot (supplemental Fig. S2) and confirmed a severalfold increase in $\mathrm{RNA}^{\mathrm{Arg} 4}$ by introduction of pArgU.

Structural Studies on the Role of $\pi m^{5} U$ in Decoding-To study the role of the taurine modification in decoding, an anticodon stem-loop containing $\tau \mathrm{m}^{5} \mathrm{U}$ at the wobble position $\left(\mathrm{ASL}_{\tau \mathrm{m} 5 \mathrm{UAA}}^{\mathrm{Leu}}\right.$ ) was constructed by enzymatic ligation of RNA fragments. ASL $\mathrm{Lm}_{\tau \mathrm{MUAA}}^{\mathrm{Leu}}$ together with one of two oligonucleotides containing one of the two leucine codons (UU(A/G)) were soaked into preformed crystals of the $30 \mathrm{~S}$ ribosomal subunit from $T$. thermophilus. The two resulting structures were solved to resolutions of between 2.5 and $2.9 \AA$ (Table 1). Surprisingly, and in contrast with the quality of the data, no defined electron density for the $5^{\prime}$ part of the ASL was visible at a high contouring level in the two structures, including U33, nor for the modification itself, indicating a high degree of flexibility (Fig. 5, $A$

TABLE 1

Data collection and refinement statistics (molecular replacement)

\begin{tabular}{|c|c|c|}
\hline & $\tau \mathrm{m}^{5} \mathrm{U}-\mathrm{A}$ & $\tau \mathrm{m}^{5} \mathrm{U}-\mathrm{G}$ \\
\hline \multicolumn{3}{|l|}{ Data collection } \\
\hline Space group & $\mathrm{P} 4_{1} 2_{1} 2$ & $\mathrm{P} 4_{1} 2_{1} 2$ \\
\hline \multicolumn{3}{|l|}{ Cell dimensions } \\
\hline$a, b, c(\AA)$ & $\begin{array}{c}a=b=402.0 \\
c=175\end{array}$ & $\begin{array}{c}a=b=402.5 \\
c=176.0\end{array}$ \\
\hline$\alpha, \beta, \gamma$ (degrees) & $\alpha=\beta=\gamma=90$ & $\alpha=\beta=\gamma=90$ \\
\hline Resolution $(\AA)$ & $50-2.5(2.6-2.5)^{a}$ & $50-2.9(3.0-2.9)^{a}$ \\
\hline$R_{\text {sym }}$ or $R_{\text {merge }}$ & $15.5(76.6)^{a}$ & $13.8(64.5)^{a}$ \\
\hline$I / \sigma I$ & $9.7(2.3)^{a}$ & $7.9(2.1)^{a}$ \\
\hline Completeness (\%) & $97.4(97.3)^{a}$ & $93.8(89.9)^{a}$ \\
\hline Redundancy & 7.5 & 4.1 \\
\hline \multicolumn{3}{|l|}{ Refinement } \\
\hline Resolution $(\AA)$ & $50-2.5(2.6-2.5)^{a}$ & $50-2.9(3.0-2.9)^{a}$ \\
\hline No. of reflections & 549,352 & 341,198 \\
\hline$R_{\text {work }} / R_{\text {free }}$ & $25.5 / 28.3$ & $22.3 / 25.5$ \\
\hline \multicolumn{3}{|l|}{ No. of atoms } \\
\hline RNA & 32,745 & 32,746 \\
\hline Protein & 19,250 & 19,250 \\
\hline Ions & 120 & 120 \\
\hline Paromomycin & 42 & 42 \\
\hline \multicolumn{3}{|l|}{$B$-factors } \\
\hline RNA & 63.43 & 66.78 \\
\hline Protein & 67.43 & 69.24 \\
\hline Ions & 61.32 & 64.67 \\
\hline Paromomycin & 55.90 & 57.83 \\
\hline \multicolumn{3}{|c|}{ Root mean square deviations } \\
\hline Bond lengths $(\AA)$ & 0.0066 & 0.0073 \\
\hline Bond angles (degrees) & 1.16 & 1.21 \\
\hline
\end{tabular}

and $B$ ). However, at low contouring level, weak electron density for the taurinomethyl group was visible in unbiased difference Fourier maps, in agreement with the final refined position of the modification. The $\mathrm{p} K_{a}$ of the sulfonic acid group of taurine is about 1.5, suggesting that it is completely deprotonated. Interestingly, the refined position of the modification places the oxygens of the sulfonic acid group within hydrogen bonding distance of the exocyclic amino groups of A35, and A36 as well as the $2^{\prime}-\mathrm{OH}$ of U33. Furthermore, the secondary amine of the modification is within hydrogen bonding distance of the $2^{\prime}-\mathrm{OH}$ of U33. However, although these potential interactions could preorder the anticodon loop and restrict the flexibility of the wobble base, they do not seem to constrain the modification or the $5^{\prime}$ part of the ASL into a defined position.

The electron density for the two base pairs is very similar. The $\tau \mathrm{m}^{5} \mathrm{U}$-A base pair refined into a position with a slightly distorted Watson-Crick geometry (Fig. $5 \mathrm{C}$ ). Interestingly the $\tau \mathrm{m}^{5} \mathrm{U} \cdot \mathrm{G}$ base pair does not appear to have standard $\mathrm{G} \cdot \mathrm{U}$ wobble geometry (Fig. $5 D$ ). This is most directly seen in Fig. $5 D$, because modeling a $\mathrm{G} \cdot \mathrm{U}$ wobble base pair in the wobble position would clearly place the uridine out of density (Fig. $5 D$ ).

\section{DISCUSSION}

Using an in vitro mitochondrial translation system, we previously examined the decoding activity of a mutant $\mathrm{mt}$ tRNA ${ }^{\text {Leu(UUR) }}$ from cells of MELAS patients that lack the $\tau \mathrm{m}^{5} \mathrm{U}$ modification and a wild-type $\mathrm{mt}$ tRNA ${ }^{\mathrm{Leu}(\mathrm{UUR})}$ whose $\tau \mathrm{m}^{5} \mathrm{U}$ modification was surgically replaced by an unmodified uridine (27). The result of this study clearly showed that $\pi \mathrm{m}^{5} \mathrm{U}$ was required for decoding the UUG codon. However, we had to use a polymer of UUR codons (poly(UUR) ${ }_{30}$ ) as an mRNA to test the decoding activity of mt tRNAs, because natural mRNAs initiating with an AUG codon are not translated by the in vitro 


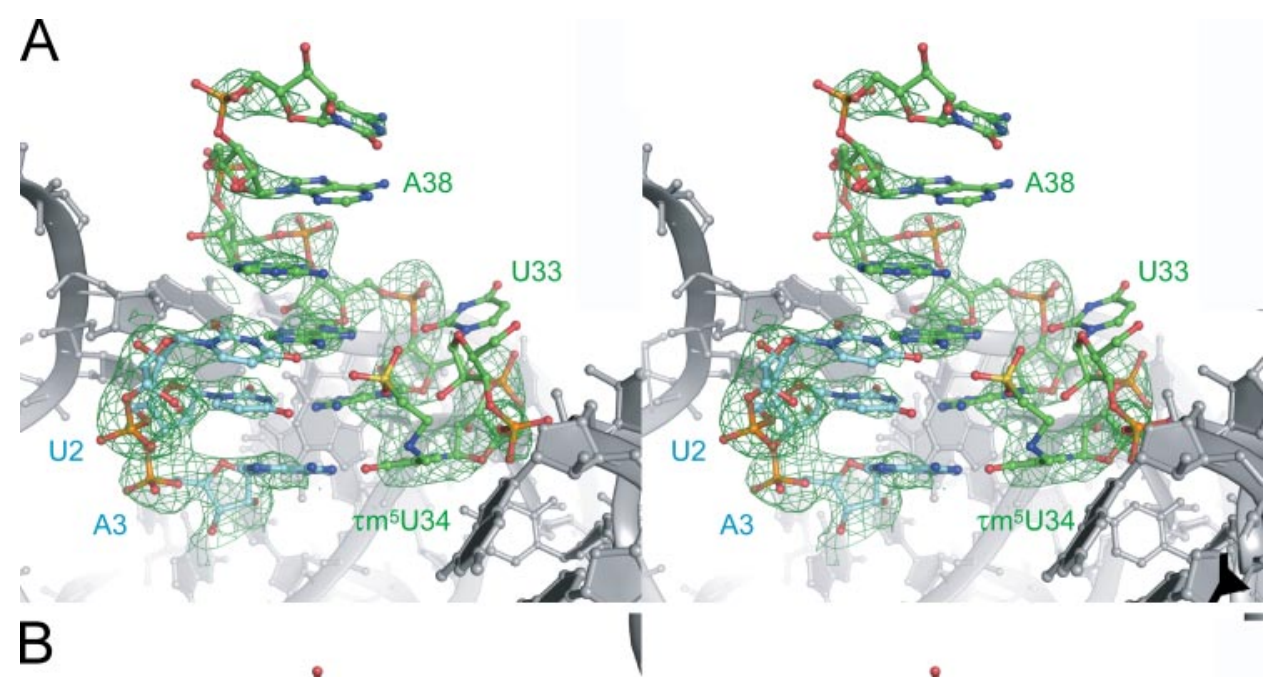

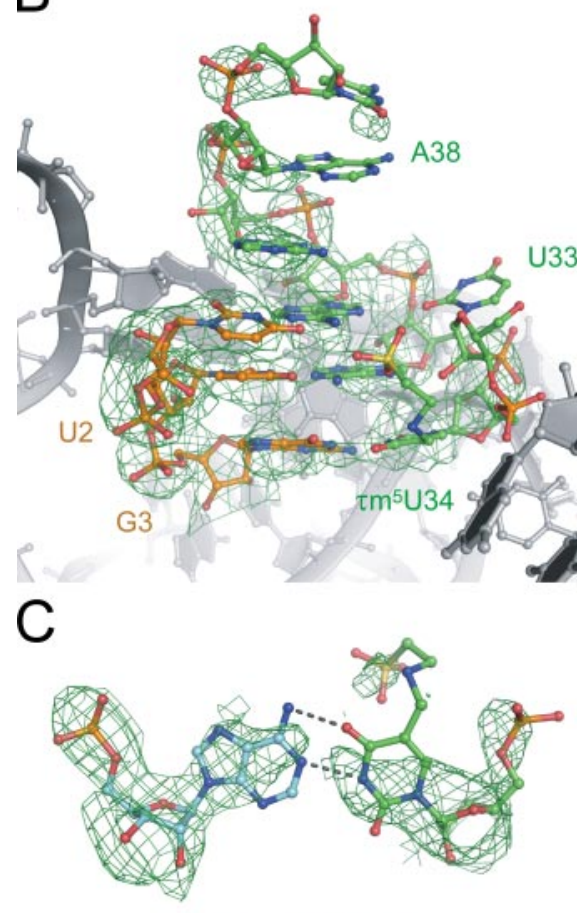

$A-\tau m^{5} U$
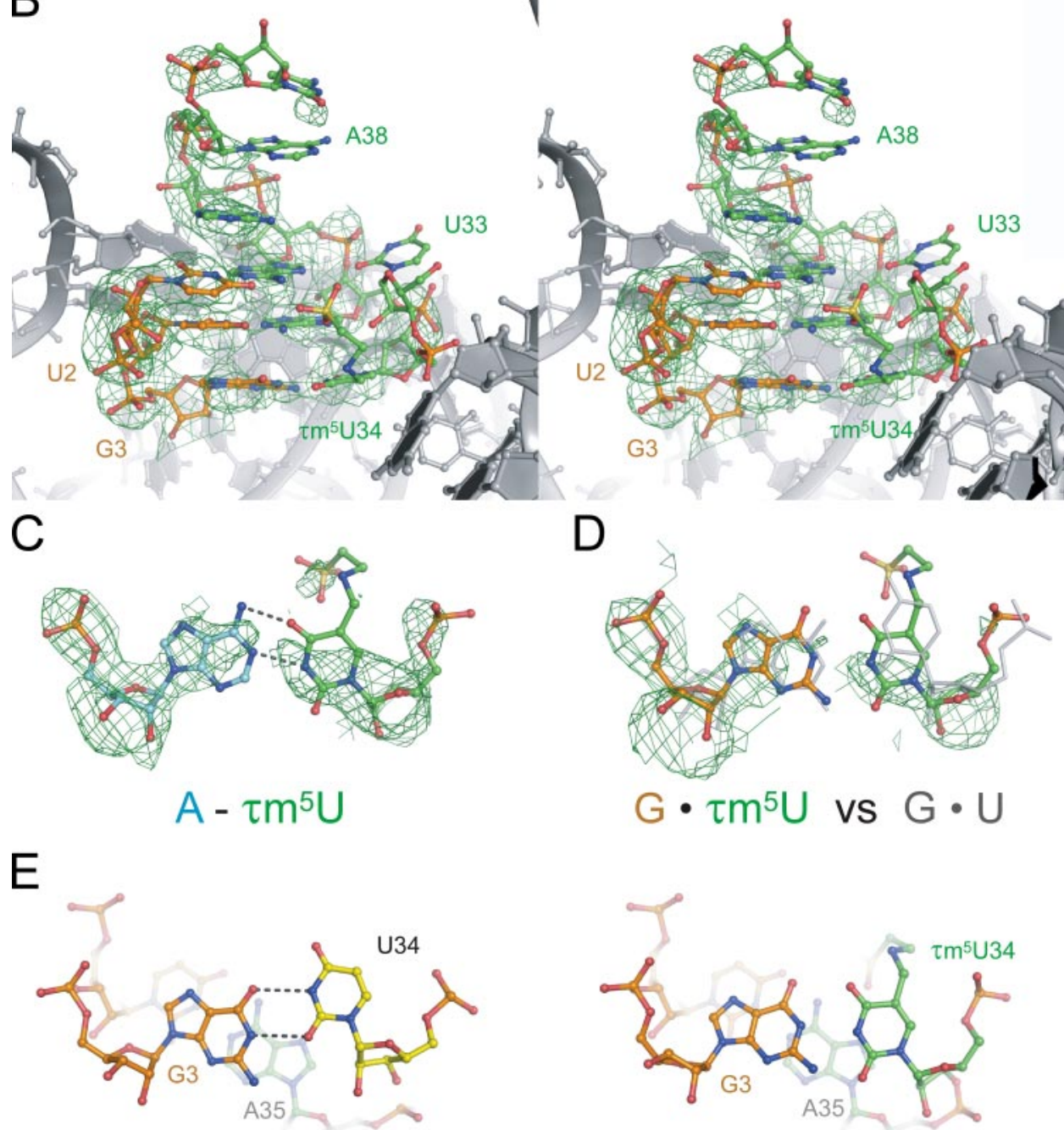

D
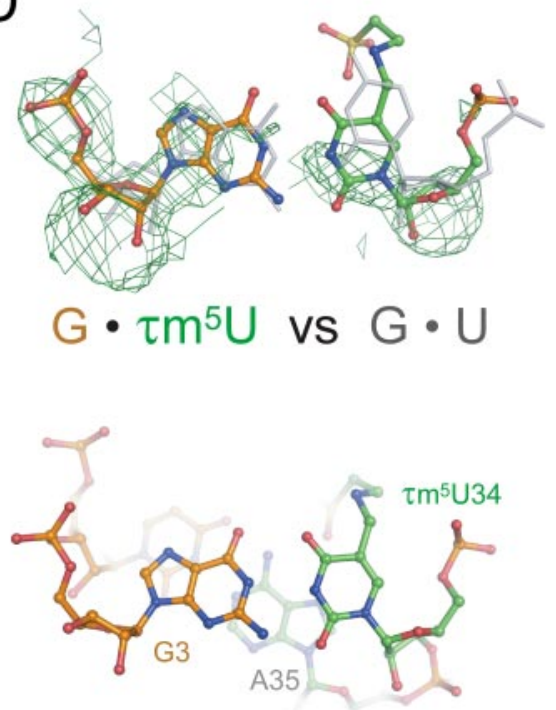

comparison of stacking interaction of $\mathrm{G} \cdot \mathrm{U}$ vs $\mathrm{G} \cdot \tau \mathrm{m}^{5} \mathrm{U}$

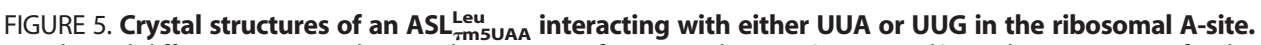
$A$, unbiased difference Fourier electron density maps for ASL and mRNA (green mesh) are shown in stereo for the complex with the UUA codon. The $5^{\prime}$ part of the ASL up to and including parts of U33 is not visible. $B$, the same as $A$ but for the complex having a UUG codon in the A-site. $C$, the $\pi \mathrm{m}^{5} \mathrm{U}-\mathrm{A}$ base pair along with unbiased difference Fourier electron density maps (green mesh). The base pair refined into a position with slightly distorted WC geometry. Weak density for the sulfonic acid group was visible at this $\sigma$ level. $D$, the $\tau \mathrm{m}^{5} \mathrm{U} \cdot \mathrm{G}$ base pair with unbiased difference Fourier electron density maps (green mesh). Although the density is not very strong, it can be excluded that the base pair adopts G.U wobble geometry (compare modeled G.U base pair in transparent gray with electron density maps).E, comparison of stacking interaction between a modeled G.U wobble base pair and the $\pi \mathrm{m}^{5} \mathrm{U} \cdot \mathrm{G}$ base pair. The modified uridine has a more favorable stacking interaction with A35 than an unmodified base would have. translation system using mitochondrial ribosomes and factors. This experimental limitation of the mitochondrial system prompted us to design a bacterial system to measure the decoding activity of tRNAs with various $\mathrm{xm}^{5} \mathrm{U}$ modifications. In addition, confirming our observations in another system further supports the hypothesis that the $\mathrm{xm}^{5} \mathrm{U}$ modification plays a critical role in decoding NNG codons by stabilizing U.G wobble pairing. By combining chemical synthesis of pcmnm ${ }^{5} U p$ and $p \pi m^{5} U p$ as monomer units and enzymatic ligation of RNA oligonucleotides, we successfully constructed E. coli tRNA ${ }^{\text {Leu(UUR) }}$ bearing $\mathrm{cmnm}^{5} \mathrm{U}$ or $\tau \mathrm{m}^{5} \mathrm{U}$ at the wobble position. Since E. coli leucyl-tRNA synthetase does not recognize wobble modifications (58), both modified and unmodified tRNAs can be efficiently aminoacylated in vitro. The A-site tRNA binding experiments revealed that tRNAs with $\mathrm{xm}^{5} \mathrm{U}$ modifications showed efficient binding to the UUG codon, whereas unmodified tRNA did not. This finding is consistent with our previous data using mt tRNA ${ }^{\mathrm{Leu}(\mathrm{UUR})}$ without the $\tau \mathrm{m}^{5} \mathrm{U}$ modification (27). By employing an in vitro E. coli cellfree translation system, we measured the decoding activities of the modified tRNAs. In this system, we used mRNAs bearing a single UUR codon and encoding a short 17-amino acid peptide (Met-Ile ${ }_{10}-\mathrm{Leu}-\mathrm{Val}_{5}$ ). The $\mathrm{cmnm}^{5}$ modification clearly enhanced UUG translation, as observed in the mitochondrial system (27). In contrast, the E. coli tRNA ${ }^{\text {Leu(UUR) }}$ with $\tau \mathrm{m}^{5} \mathrm{U}$ exhibited reduced UUA decoding, but decoding of UUG was increased almost 2 -fold (Fig. 2B). The heterologous combination of the E. coli tRNA $^{\text {Leu(UUR) }}$ with a mitochondrial modification may have resulted in a loss of decoding activity. The E. coli tRNA $^{\text {Leu(UUR) }}$ with $\tau \mathrm{m}^{5} \mathrm{U}$ did translate both codons at similar levels, suggesting that the $\tau \mathrm{m}^{5}$ modification does play a role in decoding UUG.

In this study, we observed that the E. coli tRNA ${ }^{\text {Leu(UUR) }}$ with an unmodified U34 was unable to 


\section{Wobble Modifications Stabilize U.G Pairing}

translate UUY codons, similar to our previous results using the mitochondrial translation system (27). Based on the mitochondrial four-way wobbling, a tRNA with U34 should translate NNY codons. In the mitochondrial decoding table (7), there are eight family boxes (CUN, GUN, UCN, CCN, ACN, GCN, CGN, and GGN), each of which is decoded by a single tRNA with U34. All of these boxes are decoded by tRNAs forming either two GC base pairs or one GC base pair but having a purine in position 35 of the tRNA. In contrast to a pyrimidine, a purine in position 35 can form a stronger hydrogen bond to the 2'-OH of U33, which presumably affects preordering of the anticodon loop, thereby reducing the entropic cost of binding. UUR codons have no GC pair in the first two positions. These facts strongly suggest that, if there are either two GC pairs in the first two positions or one GC pair in combination with a purine in position 35 of the tRNA, U34 can decode any base at the third position. However, if there is no GC in the first two positions, U34 can only decode NNA and NNG codons, due to weak hydrogen bonding in the codon-anticodon interaction. This study, together with our previous observations (27), indicates that $\mathrm{xm}^{5} \mathrm{U}$ wobble modifications in tRNA ${ }^{\mathrm{Leu}(U U R)}$ play a critical role in decoding UUG codons by stabilizing the U.G wobble base pair rather than preventing decoding of UUY codons. This model is consistent with our finding that $m t$ tRNA ${ }^{\text {Lys }}$, obtained from patients with the mitochondrial disease MERRF, lacks the $\tau \mathrm{m}^{5} \mathrm{~s}^{2} \mathrm{U}$ modification due to the pathogenic 8344 mutation and was unable to translate AAY codons in addition to AAG codons (59), because the AAR codons do not have $\mathrm{G}$ nor $\mathrm{C}$ in the first two positions.

We also examined the decoding properties of E.coli tRNA $^{\text {Arg4 }}$ without the $\mathrm{mnm}^{5} \mathrm{U}$ modification by pulse labeling and using fus $A$ reporters having consecutive AGR codons. We chose the AGR codon to quantify the decoding activity of $x \mathrm{~m}^{5} \mathrm{U}$ modifications in the cell, because bacterial arginyl-tRNA synthetase recognizes A20 in the D-arm and C35 (second base of the anticodon) as major determinants and does not recognize the wobble position (60). Using the $\triangle m n m E$ strain, E.coli tRNA $^{\text {Arg4 }}$ lacking $\mathrm{mnm}^{5} \mathrm{U}$ stalled at both the (AGA) $)_{3}$ and $(\mathrm{AGG})_{2}$ sites of the fusA gene, whereas E. coli tRNA ${ }^{\mathrm{Arg} 4}$ in wildtype cells showed efficient translation of (AGG) ${ }_{2}$ but still stalled at (AGA) $)_{3}$. These data reveal that the $\mathrm{mnm}^{5}$ modification of E. coli tRNA $^{\text {Arg4 }}$ is required to decode tandem AGG codons more efficiently. Although the AGG codon is redundantly decoded by two minor tRNAs bearing the $\mathrm{mnm}^{5} \mathrm{UCU}$ anticodon (tRNA ${ }^{\mathrm{Arg} 4}$ ) and $\mathrm{CCU}$ anticodon (tRNA ${ }^{\mathrm{Arg} 5}$ ), this study demonstrated that E. coli $\mathrm{tRNA}^{\mathrm{Arg} 4}$ with the $\mathrm{mnm}^{5} \mathrm{U}$ modification is actually required for efficient translation of AGG codons, whereas $\mathrm{mnm}^{5} \mathrm{U}$ does not influence AGA decoding. These data are consistent with the in vitro observations. Since ribosomal stalling at consecutive AGR codons was rescued by overexpression of E. coli tRNA ${ }^{\mathrm{Arg} 4}$, with or without the $\mathrm{mnm}^{5} \mathrm{U}$ modification, efficient decoding of NNG codons can be compensated by tRNAs with an unmodified wobble uridine, if present at high concentrations in the cell. However, misreading NNY codons due to a lack of wobble modification may occur. Thus, $\mathrm{xm}^{5} \mathrm{U}$ modifications enable minor
tRNAs to decode NNG codons by stabilizing $U \cdot G$ wobble pairing.

The decoding of GAR codons in vivo was examined using lac $Z$ reporters bearing eight consecutive GAR codons for Glu (56). The wobble position of tRNA ${ }^{\text {Glu }}$ is modified to $\mathrm{mnm}^{5} \mathrm{~s}^{2} \mathrm{U}$. The tRNA ${ }^{\text {Glu }}$ lacking the $\mathrm{mnm}^{5}$ modification but containing $\mathrm{s}^{2} \mathrm{U} 34$ translated the GAA codon more efficiently than the GAG codon. In contrast, tRNA ${ }^{\text {Glu }}$ lacking the 2-thio group but containing $\mathrm{mnm}^{5} \mathrm{U} 34$ showed less activity toward both codons, 4-fold slower for GAA and 20\% slower for GAG. These data clearly show that the $\mathrm{mnm}^{5}$ and 2-thio modifications of tRNA $^{\text {Glu }}$ have distinct functions in codon recognition and that the $\mathrm{mnm}^{5}$ modification is responsible for GAG decoding. These results are consistent with our current results for the decoding of UUR codons.

Recent structural studies of codon-anticodon interactions at the A-site of the $30 \mathrm{~S}$ ribosomal subunit partially explain the functional role of $\mathrm{xm}^{5} \mathrm{U}$ modifications. The crystal structure of an ASL containing $\mathrm{mnm}^{5} \mathrm{U}$ complexed with a short mRNA and the ribosomal $30 \mathrm{~S}$ subunit revealed a geometry distinct from the expected U.G wobble geometry, although the $\mathrm{mnm}^{5}$ modification itself was not visible in the electron density (61). In the present study, we see a similar situation for the $\tau \mathrm{m}^{5} \mathrm{U} \cdot \mathrm{G}$ base pair, for which the wobble geometry can clearly be ruled out. The question is why a classical U.G wobble base pair needs to be avoided. The third base in the codon (G in the present structure) is constrained by contacts to $16 \mathrm{~S} \mathrm{rRNA}$. This requires the uridine in the tRNA to move toward the major groove of the codon-anticodon helix by about $2.5 \AA$ in order to adopt canonical wobble geometry and would result in an almost complete loss of stacking interaction with the base in position 35 (Fig. $5 E$ ). In the $\tau \mathrm{m}^{5} \mathrm{U} \cdot \mathrm{G}$ wobble geometry, the $\tau \mathrm{m}^{5} \mathrm{U}$ is shifted toward the minor groove, thereby having a more favorable stacking interaction with the adenine in position 35 (A35). The exact nature of the base pair cannot be definitely ascertained. It could be a Watson-Crick base pair, which would require the $U$ to be in the enol form. In fact, it has been proposed that the H3 proton of $\mathrm{xm}^{5} \mathrm{U}$ tends to be deprotonated (62). However, it is not clear why this modification should induce an enol tautomer of the base. Another possibility is that the base pair is intermediate between a standard WC and the classical U.G wobble position, as observed before in the $m n m^{5} \mathrm{U} \cdot \mathrm{G}$ pair. This would result in a bifurcated hydrogen bond between $\mathrm{O} 2$ of the $\tau \mathrm{m}^{5} \mathrm{U}$ and N1/N2 of the $\mathrm{G}$, which is not as strong as two complete hydrogen bonds of the classical U.G wobble pair (63), but the uridine would still have a more favorable stacking overlap with A35. Classical U.G wobble pairs at the end of helices have a preference for the $U$ being on the $3^{\prime}$-end rather than on the $5^{\prime}$-end due to lack of base stacking (51). It is reasonable to assume that a $5^{\prime}-\mathrm{U}$ at the end of the codon-anticodon helix is energetically unfavorable. The role of the taurine modification and more generally the role of $\mathrm{xm}^{5} \mathrm{U}$ modifications could be facilitating the formation of $U \cdot G$ base pairs in which the uridine being on the $5^{\prime}$-end of the helix has a more favorable stacking interaction. Molecular interactions between the C5-substituent of $\mathrm{xm}^{5} \mathrm{U}$ and other parts of the ASL, the mRNA, or the decoding center of $16 \mathrm{~S}$ rRNA may stabilize the $\mathrm{xm}^{5} \mathrm{U} \cdot \mathrm{G}$ wobble geometry. At this point, it cannot be excluded that in 
the context of the mitochondrial ribosome, additional contacts to elements not present in the eubacterial one can be made that would put more constraints on the C5-substituent. Alternatively, the chemical characteristics of the wobble modification may influence the structure of the wobble base. Unfortunately, a direct visualization of the C5-substituent of the wobble base in the decoding center, which would provide an understanding of the functional role of $\mathrm{xm}^{5} \mathrm{U}$ modification at the atomic level, remains elusive and may require studies with the mitochondrial ribosome.

Acknowledgments-We are grateful to Takeo Suzuki, K. Miyauchi, M. Iyo, T. Ogata, S. Nakagawa, and Frank V. Murphy for technical assistance and helpful suggestions. Special thanks are due to Dr. K. Saigo (University of Tokyo) for helpful suggestions and encouragement during this project.

\section{REFERENCES}

1. Ogle, J. M., Brodersen, D. E., Clemons, W. M., Jr., Tarry, M. J., Carter, A. P., and Ramakrishnan, V. (2001) Science 292, 897-902

2. Ogle, J. M., Murphy, F. V., Tarry, M. J., and Ramakrishnan, V. (2002) Cell 111, 721-732

3. Crick, F. H. (1966) J. Mol. Biol. 19, 548-555

4. Bjork, G. R. (1995) Biosynthesis and Function of Modified Nucleosides, pp. 165-205, American Society for Microbiology Press, Washington, DC

5. Curran, J. F. (1998) Modified Nucleosides in Translation, pp. 493-516, American Society for Microbiology Press, Washington, DC

6. Yokoyama, S., and Nishimura, S. (1995) Modified Nucleosides and Codon Recognition (Soll, D., and Rajbandary, U. L. eds) pp. 207-224, American Society for Microbiology Press, Washington, DC

7. Suzuki, T. (2005) in Fine-tuning of RNA Functions by Modification and Editing, pp. 24-69, Springer-Verlag New York Inc., New York

8. Agris, P. F., Vendeix, F. A., and Graham, W. D. (2007) J. Mol. Biol. 366, $1-13$

9. Bonitz, S. G., Berlani, R., Coruzzi, G., Li, M., Macino, G., Nobrega, F. G., Nobrega, M. P., Thalenfeld, B. E., and Tzagoloff, A. (1980) Proc. Natl. Acad. Sci. U. S. A. 77, 3167-3170

10. Barrell, B. G., Anderson, S., Bankier, A. T., de Bruijn, M. H., Chen, E., Coulson, A. R., Drouin, J., Eperon, I. C., Nierlich, D. P., Roe, B. A., Sanger, F., Schreier, P. H., Smith, A. J., Staden, R., and Young, I. G. (1980) Proc. Natl. Acad. Sci. U. S. A. 77, 3164-3166

11. Andachi, Y., Yamao, F., Iwami, M., Muto, A., and Osawa, S. (1987) Proc. Natl. Acad. Sci. U. S. A. 84, 7398-7402

12. Inagaki, Y., Kojima, A., Bessho, Y., Hori, H., Ohama, T., and Osawa, S. (1995) J. Mol. Biol. 251, $486-492$

13. Samuelsson, T., Elias, P., Lustig, F., Axberg, T., Folsch, G., Akesson, B., and Lagerkvist, U. (1980) J. Biol. Chem. 255, 4583-4588

14. Phelps, S. S., Malkiewicz, A., Agris, P. F., and Joseph, S. (2004) J. Mol. Biol. 338, $439-444$

15. Nasvall, S. J., Chen, P., and Bjork, G. R. (2004) RNA 10, 1662-1673

16. Sorensen, M. A., Elf, J., Bouakaz, E., Tenson, T., Sanyal, S., Bjork, G. R., and Ehrenberg, M. (2005) J. Mol. Biol. 354, 16-24

17. Martin, R. P., Sibler, A. P., Gehrke, C. W., Kuo, K., Edmonds, C. G., McCloskey, J. A., and Dirheimer, G. (1990) Biochemistry 29, $956-959$

18. Andachi, Y., Yamao, F., Muto, A., and Osawa, S. (1989) J. Mol. Biol. 209, $37-54$

19. Yokoyama, S., Watanabe, T., Murao, K., Ishikura, H., Yamaizumi, Z., Nishimura, S., and Miyazawa, T. (1985) Proc. Natl. Acad. Sci. U. S. A. 82, 4905-4909

20. Watanabe, K., and Osawa, S. (1995) tRNA Sequence and Variations in the Genetic Code, pp. 225-250, American Society for Microbiology Press, Washington, DC

21. Suzuki, T., Suzuki, T., Wada, T., Saigo, K., and Watanabe, K. (2002) EMBO
J. 21, 6581-6589

22. Yasukawa, T., Suzuki, T., Suzuki, T., Ueda, T., Ohta, S., and Watanabe, K. (2000) J. Biol. Chem. 275, 4251-4257

23. Yasukawa, T., Suzuki, T., Ishii, N., Ueda, T., Ohta, S., and Watanabe, K. (2000) FEBS Lett. 467, 175-178

24. Kirino, Y., Goto, Y., Campos, Y., Arenas, J., and Suzuki, T. (2005) Proc. Natl. Acad. Sci. U. S. A. 102, 7127-7132

25. Kirino, Y., and Suzuki, T. (2005) RNA Biol. 2, 41-44

26. Yasukawa, T., Kirino, Y., Ishii, N., Holt, I. J., Jacobs, H. T., Makifuchi, T., Fukuhara, N., Ohta, S., Suzuki, T., and Watanabe, K. (2005) FEBS Lett. 579, $2948-2952$

27. Kirino, Y., Yasukawa, T., Ohta, S., Akira, S., Ishihara, K., Watanabe, K., and Suzuki, T. (2004) Proc. Natl. Acad. Sci. U. S. A. 101, 15070-15075

28. Malkiewicz, A., Sochacka, E., Sayed Ahmed, A. F., and Yassin, S. (1983) Tetrahedron Lett. 24, 5395-5398

29. Murao, K., and Ishikura, H. (1978) Nucleic Acids Res. Special Publication 5, (suppl.) s333-s336

30. Barrio, J. R., Barrio, M. C., Leonard, N. J., England, T. E., and Uhlenbeck, O. C. (1978) Biochemistry 17, 2077-2081

31. Kurata, S., Ohtsuki, T., Suzuki, T., and Watanabe, K. (2003) Nucleic Acids Res. 31, e145

32. Ohtsuki, T., Kawai, G., and Watanabe, K. (1998) J. Biochem. (Tokyo) 124, $28-34$

33. Suzuki, T., Ueda, T., and Watanabe, K. (1997) EMBO J. 16, 1122-1134

34. Donis-Keller, H. (1980) Nucleic Acids Res. 8, 3133-3142

35. Noma, A., Kirino, Y., Ikeuchi, Y., and Suzuki, T. (2006) EMBO J. 25, 2142-2154

36. Suzuki, T., Ikeuchi, Y., Noma, A., Suzuki, T., and Sakaguchi, Y. (2007) Methods Enzymol. 425, 211-229

37. Kaneko, T., Suzuki, T., Kapushoc, S. T., Rubio, M. A., Ghazvini, J., Watanabe, K., Simpson, L., and Suzuki, T. (2003) EMBO J. 22, 657-667

38. Ashraf, S. S., Sochacka, E., Cain, R., Guenther, R., Malkiewicz, A., and Agris, P. F. (1999) RNA 5, $188-194$

39. Spedding, G. (ed) (1990) Ribosomes and Protein Synthesis: A Practical Approach, pp. 1-30, Oxford University Press, New York

40. Takai, K., Takaku, H., and Yokoyama, S. (1996) Nucleic Acids Res. 24, $2894-2899$

41. Milligan, J. F., and Uhlenbeck, O. C. (1989) Methods Enzymol. 180, 51-62

42. Datsenko, K. A., and Wanner, B. L. (2000) Proc. Natl. Acad. Sci. U. S. A. 97, $6640-6645$

43. Varenne, S., Knibiehler, M., Cavard, D., Morlon, J., and Lazdunski, C. (1982) J. Mol. Biol. 159, 57-70

44. Tomita, K., Ogawa, T., Uozumi, T., Watanabe, K., and Masaki, H. (2000) Proc. Natl. Acad. Sci. U. S. A. 97, $8278-8283$

45. Clemons, W. M., Jr., Brodersen, D. E., McCutcheon, J. P., May, J. L., Carter, A. P., Morgan-Warren, R. J., Wimberly, B. T., and Ramakrishnan, V. (2001) J. Mol. Biol. 310, 827-843

46. Kabsch, W. (1993) J. Appl. Crystallogr. 26, 795-800

47. Collaborative Computational Project 4 (1994) Acta Crystallogr. D Biol. Crystallogr. 50, 760-763

48. Emsley, P., and Cowtan, K. (2004) Acta Crystallogr. D Biol. Crystallogr. 60, $2126-2132$

49. Brunger, A. T., Adams, P. D., Clore, G. M., DeLano, W. L., Gros, P., Grosse-Kunstleve, R. W., Jiang, J. S., Kuszewski, J., Nilges, M., Pannu, N. S., Read, R. J., Rice, L. M., Simonson, T., and Warren, G. L. (1998) Acta Crystallogr. D Biol. Crystallogr. 54, 905-921

50. Kleywegt, G. J., and Jones, T. A. (1998) Acta Crystallogr. D Biol. Crystallogr. 54, 1119-1131

51. Mizuno, H., and Sundaralingam, M. (1978) Nucleic Acids Res. 5, $4451-4461$

52. Masquida, B., Sauter, C., and Westhof, E. (1999) RNA 5, 1384-1395

53. Spanjaard, R. A., Chen, K., Walker, J. R., and van Duin, J. (1990) Nucleic Acids Res. 18, 5031-5036

54. Chen, G. T., and Inouye, M. (1994) Genes Dev. 8, 2641-2652

55. Rosenberg, A. H., Goldman, E., Dunn, J. J., Studier, F. W., and Zubay, G. (1993) J. Bacteriol. 175, 716-722

56. Kruger, M. K., Pedersen, S., Hagervall, T. G., and Sorensen, M. A. (1998) J. Mol. Biol. 284, 621-631 
57. Hagervall, T. G., Edmonds, C. G., McCloskey, J. A., and Bjork, G. R. (1987) J. Biol. Chem. 262, $8488-8495$

58. Asahara, H., Himeno, H., Tamura, K., Hasegawa, T., Watanabe, K., and Shimizu, M. (1993) J. Mol. Biol. 231, 219-229

59. Yasukawa, T., Suzuki, T., Ishii, N., Ohta, S., and Watanabe, K. (2001) EMBO J. 20, 4794-4802
60. Shimada, A., Nureki, O., Goto, M., Takahashi, S., and Yokoyama, S. (2001) Proc. Natl. Acad. Sci. U. S. A. 98, 13537-13542

61. Murphy, F. V., IV, Ramakrishnan, V., Malkiewicz, A., and Agris, P. F. (2004) Nat. Struct. Mol. Biol. 11, 1186-1191

62. Takai, K., and Yokoyama, S. (2003) Nucleic Acids Res. 31, 6383-6391

63. Yang, J., and Gellman, S. H. (1998) J. Am. Chem. Soc. 120, 9090-9091 